Pacific Journal of Mathematics

WEAK APPROXIMATION OF STRATEGIES IN MEASURABLE 


\title{
WEAK APPROXIMATION OF STRATEGIES IN MEASURABLE GAMBLING
}

\author{
Victor C. Pestien
}

In a measurable gambling house, the measurable strategies available at a fortune $f$ are weakly dense in the collection of all strategies available at $f$.

1. Background. In $\$ 2.16$ of their monograph on the theory of gambling, Dubins and Savage [3] showed how the imposition of regularity conditions on a gambling problem yields regularity properties about its solution. In particular, they established certain continuity conditions which guarantee the existence of Borel-measurable, nearly-optimal strategies. Strauch [9] defined the notion of measurable gambling house and showed that if a measurable house is leavable, then the optimal return function is universally measurable and good measurable strategies exist. Without assuming leavability, Sudderth [10] investigated measurable gambling problems and showed, among other things, that if the gambler's payoff, or utility, under a strategy $\hat{\sigma}$ is $\int g d \sigma$, where $g$ is a bounded, finitary function, then the gambler may, without loss, restrict himself to measurable strategies.

However, the question of adequacy of measurable strategies for measurable problems - with a strategic utility function of the type introduced by Dubins and Savage - remained unanswered. (See remarks in [5].) Recently, though, Purves and Sudderth [8] established certain approximation results which provide a greater understanding of the optimal return function and, at least in some cases, imply its universal measurability.

In this note, we clarify the position of the set of measurable strategies available at a fortune $f$ within the set of all strategies available at $f$ by showing that the former set is "weakly dense" in the latter. This density property, however, is not strong enough to answer the question of adequacy of measurable strategies.

We avoid entirely any discussion of optimality and, instead, examine the strategic measures directly. We demonstrate that if $A_{1}, A_{2}, \ldots, A_{n}$ are finitary, Borel subsets of the space of histories, and if $\sigma$ is an available strategy, then there is an available measurable strategy $\hat{\sigma}$ which makes 
$\left|\sigma\left(A_{h}\right)-\hat{\sigma}\left(A_{h}\right)\right|$ simultaneously small for each $k, 1 \leq k \leq n$. Precise definitions follow in the next section.

2. Terminology. Let $F$ (the fortune space) be a Polish space and 9 be the $\sigma$-algebra of its Borel subsets. Let $P_{F}$ be the set of all countably additive probability measures defined on $(F, \Re)$, with the weak topology. To each element $f$ of $F$ assign a non-empty set $\Gamma(f)$ of gambles (finitely additive probability measures defined on all subsets of $F$ ), and let $p(\gamma)$ denote the restriction of a gamble $\gamma$ to 93 . Assume that $\Gamma$ is a Borel gambling house; that is, for every $f \in F$ and $\gamma \in \Gamma(f), p(\gamma)$ is countably additive and $\{(f, p(\gamma)): \gamma \in \Gamma(f)\}$ is a Borel subset of $F \times P_{F}$. This definition of Borel gambling house is the same as that of "measurable gambling house" in [9].

Let $H$, the space of histories, be the Cartesian product of countably many copies of the Polish space $F$. Designate the product topology on $H$ by $\Pi$. Also define a second topology, $\Delta$, on $H$ by ignoring the Polish-space structure of $F$ and letting $\Delta$ be the product topology on $H$ induced by giving $F$ the discrete topology.

Let $f \in F$. A strategy available at $f$ is a sequence

$$
\sigma=\left(\sigma_{0}, \sigma_{1}, \sigma_{2}, \ldots\right)
$$

where $\sigma_{0} \in \Gamma(f)$ and for $n \geq 1, \sigma_{n}$ is a map from $F^{n}$ to gambles on $F$ such that for each $\left(f_{1}, f_{2}, \ldots, f_{n}\right) \in F^{n}, \sigma_{n}\left(f_{1}, f_{2}, \ldots, f_{n}\right)$ lies in $\Gamma\left(f_{n}\right)$. A strategy $\sigma$ is $Q$-measurable if for each positive integer $n$, the map

$$
\left(f_{1}, \ldots, f_{n}\right) \rightarrow p\left(\sigma_{n}\left(f_{1}, \ldots, f_{n}\right)\right)
$$

is measurable with respect to the $\sigma$-algebra generated by the analytic subsets of $F^{n}$. An $Q$-measurable strategy $\sigma$ induces a countably additive probability measure $p(\sigma)$ on the $\Pi$-Borel subsets of $H$ (see [1], Prop. 7.45 and Cor. 7.42.1). Further, as described in ([3], Chapter 2), any strategy $\sigma$ determines a finitely additive probability measure (also denoted by $\sigma$ ) on the $\Delta$-clopen, or finitary, subsets of $H$. It follows from $([\mathbf{1 0}], \S 2)$, or is easily seen by induction, that if $\sigma$ is an $\mathscr{Q}$-measurable strategy, then $p(\sigma)$ coincides, on the finitary II-Borel sets, with the measure described in ([3], Chapter 2).

3. The Main Theorem. Let $\Re$ be the set of finitely additive probability measures on the algebra of finitary $\Pi$-Borel subsets of $H$. For any positive integer $n$, any $\mu$ in $\Re$ and any finitary $\Pi$-Borel sets 
$A_{1}, A_{2}, \ldots, A_{n}$, let

$$
\begin{aligned}
& N\left(\mu, A_{1}, A_{2}, \ldots, A_{n}, \varepsilon\right) \\
& \quad=\left\{\mu^{\prime} \in \mathfrak{M}:\left|\mu^{\prime}\left(A_{k}\right)-\mu\left(A_{k}\right)\right|<\varepsilon \text { for all } k, 1 \leq k \leq n\right\} .
\end{aligned}
$$

It is routinely verified that the sets of the form $N\left(\mu, A_{1}, A_{2}, \ldots, A_{n}, \varepsilon\right)$ form a neighborhood system for a weak topology on $\mathfrak{T}$.

In the theorem to follow, we identify a strategy with the probability measure it induces, restricted to the finitary П-Borel sets.

THEOREM 1. Let $f$ lie in $F$ and let $\sigma$ be a strategy available at $f$ in the Borel gambling house $\Gamma$. Then $\sigma$ lies in the weak closure of the set of Q-measurable strategies available at $f$.

The proof of Theorem 1 is preceded by four lemmas; Lemma 1 is a well-known measure-theoretic fact, Lemma 2 is a refinement of Lemma 1, and Lemmas 3 and 4 are garnered from the literature of the theory of gambling. An early version of Theorem 1 appeared in ([6], Chapter 5).

Suppose $G \in \Re^{3}$. A partition of $G$ is a finite collection $\left(A_{1}, \ldots, A_{n}\right)$ of disjoint subsets of $G$ such that $\cup_{k=1}^{n} A_{k}=G$. A Borel partition of $G$ is a partition $\left(A_{1}, \ldots, A_{n}\right)$ of $G$ such that each $A_{k}(1 \leq k \leq n)$ is Borel. A Borel cover of $G$ is a finite collection of Borel subsets of $G$ whose union is $G$. A countably-additive measure $\lambda$ on the Borel subsets of $G$ is non-atomic if $\lambda(\{f\})=0$ for each element $f$ in $G$.

Lemma 1. Let $G \in \Re$, and let $\lambda$ be a finite, non-atomic, countably-additive measure on the Borel subsets of $G, n$ be a positive integer, and $\eta_{1}, \eta_{2}, \ldots, \eta_{n}$ be non-negative real numbers such that $\sum_{k=1}^{n} \eta_{k}=\lambda(G)$. Then there exists a Borel partition $\left(B_{1}, \ldots, B_{n}\right)$ of $G$ such that for each $k$, $\lambda\left(B_{k}\right)=\eta_{k}$.

Proof. The lemma is a direct consequence of Lyapunov's Convexity Theorem. For details, see ([4], §5).

LEMma 2. Let $G \in \Re$, and let $\lambda$ be a finitely-additive measure, defined on all subsets of $G$, whose restriction to the Borel subsets of $G$ is countablyadditive. If $\left(S_{1}, \ldots, S_{n}\right)$ is a partition of $G$ and $\left(C_{1}, \ldots, C_{n}\right)$ is a Borel cover of $G$ such that $S_{k} \subseteq C_{k}$ for all $k$, then there exists a Borel partition $\left(B_{1}, \ldots, B_{n}\right)$ of $G$ such that for all $k, B_{k} \subseteq C_{k}$ and $\lambda\left(B_{k}\right)=\lambda\left(S_{k}\right)$. 
Proof. Assume temporarily that the restriction of $\lambda$ to the Borel subsets of $G$ is non-atomic. Begin by converting the Borel cover $\left(C_{1}, \ldots, C_{n}\right)$ to a Borel partition: let $\vec{j}=\left(j_{1}, \ldots, j_{n}\right)$ be a binary $n$-tuple (that is, $j_{k}=0$ or $j_{k}=1$ for each $k$ ), and let

$$
D_{J}=C_{1}^{j_{1}} \cap C_{2}^{j_{2}} \cap \cdots \cap C_{n}^{j_{n}},
$$

where for each $k$,

$$
C_{k}^{J_{k}}= \begin{cases}C_{k} & \text { if } j_{k}=1, \\ G \backslash C_{k} & \text { if } j_{k}=0 .\end{cases}
$$

The collection of sets $D_{\vec{j}}$, as $\vec{j}$ ranges over binary $n$-tuples, forms a Borel partition of $G$.

Now subdivide each $D_{\vec{J}}$ in the appropriate proportions, as follows: Using Lemma 1 , for each binary $n$-tuple $\vec{j}$, obtain a Borel partition $\left(B_{1}^{\vec{j}}, \ldots, B_{n} \vec{j}\right)$ of $D_{\vec{j}}$ such that for each $k(1 \leq k \leq n)$,

$$
\lambda\left(B_{k}^{\vec{j}}\right)=\lambda\left(D_{\vec{j}} \cap S_{k}\right)
$$

and such that $B_{k}^{\vec{j}}=\varnothing$ for those $k$ where $j_{k}=0$. (Notice that $D_{\vec{j}} \cap S_{k}=\varnothing$ for those $k$ where $j_{k}=0$, because by hypothesis, $S_{k} \subseteq C_{k}$.)

Next, for each $k$, let $B_{k}$ be the union of all $B_{k}^{\vec{j}}$ as $\vec{j}$ ranges over all binary $n$-tuples. Then $\left(B_{1}, \ldots, B_{n}\right)$ is a Borel partition of $G$, and for each $k, B_{k} \subseteq C_{k}$ and $\lambda\left(B_{k}\right)=\lambda\left(S_{k}\right)$. This completes the proof of the lemma, under the extra assumption of non-atomicity.

For the proof of the general case, let $E_{k}$ consist of those $\lambda$-atoms that lie in $S_{k}$; that is,

$$
E_{k}=\bigcup\left\{f \in G: \lambda(\{f\})>0 \text { and } f \in S_{k}\right\} .
$$

Also, let $E=\cup_{k=1}^{n} E_{k}$. Then apply the preceding argument to the Borel cover $\left(C_{1} \backslash E, \ldots, C_{n} \backslash E\right)$ of $G \backslash E$ to obtain a Borel partition $\left(\hat{B}_{1}, \ldots, \hat{B}_{n}\right)$ of $G \backslash E$ with $\hat{B}_{k} \subseteq C_{k} \backslash E$ and $\lambda\left(\hat{B}_{k}\right)=\lambda\left(S_{k} \backslash E\right)$ for all $k$. Finally, let $B_{k}=\hat{B}_{k} \cup E_{k}$, and $\left(B_{1}, \ldots, B_{n}\right)$ is the desired partition of $G$.

As in [5], let

$$
\Gamma^{\infty}(f)=\{\sigma: \sigma \text { is an } \mathscr{Q} \text {-measurable strategy available at } f\} .
$$

Let $P_{H}$ be the set of all countably additive probability measures on the $\Pi$-Borel subsets of $H$; endow $P_{H}$ with the weak topology. Then the space $P_{H}$, as well as the space $P_{F}$ defined earlier, is a Polish space ([1], Prop. 7.20, 7.23). If $A \subseteq H$ and $f \in F$, let $A f$ be the set of all histories $\left(f_{1}, f_{2}, f_{3}, \ldots\right)$ in $H$ such that $\left(f, f_{1}, f_{2}, \ldots\right) \in A$. 
Lemma 3 ([5], Lemma 4.1).

$$
\left\{(f, p(\hat{\boldsymbol{\sigma}})): \hat{\boldsymbol{\sigma}} \in \Gamma^{\infty}(f)\right\}
$$

is a Borel subset of $F \times P_{H}$.

Lemma 4. If $A$ is a $\Pi$-Borel subset of $H$, then

$$
(f, p(\hat{\boldsymbol{\sigma}})) \rightarrow \hat{\boldsymbol{\sigma}}(A f)
$$

is a Borel-measurable function from $F \times P_{H}$ to $\mathbf{R}$.

Proof. Apply the lemma in $([\mathbf{1 0}], \S 5)$.

In the proof of the theorem, we employ the notions of conditional strategy $(\sigma[f])$, structure of a finitary mapping, and integral of a bounded, finitary mapping as presented in [3].

Proof of Theorem 1. Fix $n$ a positive integer. We must show:

For each $f$ in $F$, each strategy $\sigma$ available at $f$, each $\varepsilon>0$ and each $n$-tuple $\left(A_{1}, \ldots, A_{n}\right)$ of finitary $\Pi$-Borel subsets of $H$, there exists an $\Theta$-measurable strategy $\hat{\sigma}$ available at $f$ such that for each $k$ between 1 and $n$,

$$
\left|\hat{\sigma}\left(A_{k}\right)-\sigma\left(A_{k}\right)\right|<\varepsilon .
$$

We demonstrate (3.1) by transfinite induction on the maximum structure of the indicators of the finitary events $A_{1}, \ldots, A_{n}$. Assume (3.1) holds for all $f, \sigma, \varepsilon$, and all $A_{1}, \ldots, A_{n}$ such that the maximum structure of the indicators of $A_{1}, \ldots, A_{n}$ is less than the ordinal number $\alpha$. Next, let $f_{0} \in F$, $\varepsilon>0$, let $\sigma$ be available at $f_{0}$, and suppose the maximum structure of $A_{1}, \ldots, A_{n}$ is $\alpha$. Let $m$ be a positive integer such that $2 / m<\varepsilon$. For each $n$-tuple $\left(j_{1}, \ldots, j_{n}\right)$ of integers such that $0 \leq j_{k} \leq m$ for all $k, 1 \leq k \leq n$, let

$$
\begin{array}{r}
S_{\left(j_{1}, \ldots, j_{n}\right)}=\left\{f \in F: j_{k} / m \leq \sigma[f]\left(A_{k} f\right)<\left(j_{k}+1\right) / m\right. \\
\text { for each } k, 1 \leq k \leq n\} .
\end{array}
$$

The sets $S_{\left(J_{1}, \ldots, J_{n}\right)}$ form a partition of $F$. Let

$$
\begin{array}{r}
W_{\left(J_{1}, \ldots, J_{n}\right)}=\left\{(f, p(\hat{\boldsymbol{\sigma}})): \hat{\boldsymbol{\sigma}} \in \Gamma^{\infty}(f) \text { and for each } k,\right. \\
\left.\left(j_{k}-1\right) / m<\hat{\sigma}\left(A_{k} f\right)<\left(j_{k}+1\right) / m\right\}
\end{array}
$$


and let $\hat{C}_{\left(j_{1}, \ldots j_{n}\right)}$ be the projection of $W_{\left(j_{1}, \ldots, j_{n}\right)}$ onto the first coordinate. By inductive hypothesis,

$$
\hat{C}_{\left(j_{1}, \ldots, J_{n}\right)} \supseteq S_{\left(j_{1}, \ldots j_{n}\right)} \text {. }
$$

Applying Lemmas 3 and 4, $W_{\left(\jmath_{1}, \ldots, J_{n}\right)}$ is a Borel set. Then $\hat{C}_{\left(\jmath_{1} \ldots, J_{n}\right)}$, being the projection of a Borel set, is analytic and hence measurable with respect to the completion of $\sigma_{0}([\mathbf{1}], \S 7.6 .2)$. Thus there exists a Borel cover

$$
\left\{C_{\left(j_{1}, \ldots, l_{n}\right)}: 0 \leq j_{1} \leq m, \ldots, 0 \leq j_{n} \leq m\right\}
$$

such that $\sigma_{0}\left(\hat{C}_{\left(J_{1}, \ldots, j_{n}\right)}\right)=\sigma_{0}\left(C_{\left(j_{1} \ldots, j_{n}\right)}\right)$ and $C_{\left(j_{1} \ldots, J_{n}\right)} \supseteq \hat{C}_{\left(J_{1}, \ldots, j_{n}\right)}$ for each $n$-tuple $\left(j_{1}, \ldots, j_{n}\right)$. By Lemma 2 , there is a Borel partition

$$
\left\{B_{\left(\mu_{1}, \ldots, J_{n}\right)}: 0 \leq j_{1} \leq m, \ldots, 0 \leq j_{n} \leq m\right\}
$$

such that

$$
\sigma_{0}\left(B_{\left(/_{1}, \ldots, l_{n}\right)}\right)=\sigma_{0}\left(S_{\left(/_{1}, \ldots, j_{n}\right)}\right)
$$

and

$$
B_{\left(j_{1}, \ldots, j_{n}\right)} \subseteq C_{\left(j_{1}, \ldots, j_{n}\right)}
$$

for each $n$-tuple $\left(j_{1}, \ldots, j_{n}\right)$.

Using the Jankov-von Neumann Selection Theorem ([1], Prop. 7.49), for each $\left(j_{1}, \ldots, j_{n}\right)$ there is an $Q$-measurable map $\psi_{\left(j_{1}, \ldots, j_{n}\right)}: F \rightarrow P_{H}$ such that if $f \in \hat{C}_{\left(\mu_{1} \ldots, j_{n}\right)}$, then

$$
\left(f, \psi_{\left(J_{1}, \ldots, J_{n}\right)}(f)\right) \in W_{\left(J_{1}, \ldots, j_{n}\right)} .
$$

We are now in a position to define an $Q$-measurable strategy $\hat{\sigma}$ which is available at $f_{0}$ and which approximates $\sigma$; let

$$
\hat{\sigma}_{0}=\sigma_{0}
$$

and

$$
\hat{\sigma}[f]=\psi_{\left(J_{1} \ldots \ldots j_{n}\right)}(f)
$$

whenever $f \in B_{\left(1, \ldots, l_{n}\right)}$. 
Fix $k$ between 1 and $n$. Employing Theorem 2.8.1 of [3], calculate:

$$
\begin{aligned}
& \hat{\sigma}\left(A_{k}\right)=\int \hat{\sigma}[f]\left(A_{k} f\right) d \sigma_{0}(f) \\
& =\sum_{I_{n}=0}^{m} \ldots \sum_{\lambda_{1}=0}^{m} \int_{B_{\left(J_{1} \ldots . / n\right)}} \hat{\sigma}[f]\left(A_{k} f\right) d \sigma_{0}(f) \\
& \leq \sum_{J_{n}=0}^{m} \cdots \sum_{j_{1}=0}^{m} \frac{j_{h}+1}{m} \sigma_{0}\left(B_{\left(,_{1}, \ldots, j_{n}\right)}\right) \\
& =\frac{1}{m}+\sum_{j_{n}=0}^{m} \cdots \sum_{j_{1}=0}^{m} \frac{j_{k}}{m} \sigma_{0}\left(S_{\left(j_{1}, \ldots,,_{n}\right)}\right) \\
& \leq \frac{1}{m}+\sum_{J_{n}=0}^{m} \cdots \sum_{\lambda_{1}=0}^{m} \int_{S_{(/ 1, \ldots / n)}} \sigma[f]\left(A_{h} f\right) d \sigma_{0} \\
& =\frac{1}{m}+\sigma\left(A_{k}\right) \text {. }
\end{aligned}
$$

Analogously,

$$
\begin{aligned}
\hat{\sigma}\left(A_{h}\right) & \geq \sum_{I_{n}=0}^{m} \cdots \sum_{I_{1}=0}^{m} \frac{j_{k}-1}{m} \sigma_{0}\left(B_{\left(J_{1} \ldots, j_{n}\right)}\right) \\
& \geq-\frac{2}{m}+\sum_{I_{n}=0}^{m} \cdots \sum_{\lambda_{1}=0}^{m} \int_{S_{\left(/, \ldots, I_{n}\right)}} \sigma[f]\left(A_{k} f\right) d \sigma_{0}(f) \\
& =-\frac{2}{m}+\sigma\left(A_{h}\right) .
\end{aligned}
$$

We conclude

$$
\left|\hat{\sigma}\left(A_{k}\right)-\sigma\left(A_{h}\right)\right| \leq 2 / m<\varepsilon .
$$

4. Remarks and Extensions. Theorem 1 shows that one can find a measurable strategy available at $f$ which "does nearly as well as" $\sigma$ simultaneously on each of the sets $A_{1}, \ldots, A_{n}$. A theorem of Sudderth ([10], Theorem 6.4) implied that this phenomenon held in the special case where $n=1$. We paraphrase Sudderth's theorem as a corollary:

COROLlaRy. If $g$ is a bounded, finitary, and П-Borel measurable function, then for each $f$ in $F$,

$$
\begin{aligned}
& \sup \left\{\int g d \sigma: \sigma \text { is a strategy available at } f\right\} \\
& \quad=\sup \left\{\int g d \hat{\sigma}: \hat{\sigma} \text { is an } Q \text {-measurable strategy available at } f\right\} .
\end{aligned}
$$


Proof. Approximate $g$ by a simple function and apply Theorem 1 .

If $\sigma$ is a strategy, then following Dubins [2], the restriction of the inner measure of $\sigma$ to the $\Delta$-open sets is additive and hence can be extended in a unique way to a finitely additive probability measure $\sigma_{L}$ on the algebra generated by the $\Delta$-open sets. Let $\mathscr{K}$ be the algebra of sets which can be approximated from outside by an open set and from the inside by a closed set in such a way that the $\sigma_{L}$-measure of their difference is small. Denote the natural extension of $\sigma_{L}$ to $\mathscr{K}$ by $\tilde{\sigma}_{L}$. Purves and Sudderth [7] have shown that the $\Delta$-Borel subsets of $H$ lie within $\mathcal{K}$. If $B$ is an arbitrary $\Pi$-Borel (and hence $\Delta$-Borel) subset of $H$, and $\varepsilon>0$, the author does not know whether there exists an available measurable strategy $\hat{\sigma}$ such that $\left|\hat{\sigma}(B)-\tilde{\sigma}_{L}(B)\right|<\varepsilon$. Does the fact that $\sigma$ can be approximated weakly by measurable strategies imply that $\tilde{\sigma}_{L}(B)$ can be approximated in an appropriate way? The answer is not clear, even in the particular case where $B=A \times A \times A \times \cdots$, and $A$ is a Borel subset of $F$.

Acknowledgment. The author is deeply grateful to Professor Lester Dubins for providing guidance and suggestions on this topic.

\section{REFERENCES}

[1] Dimitri P. Bertsekas, and Steven E. Shreve, Stochastic Optimal Control: the Discrete Time Case, Academic Press, New York (1978).

[2] Lester E. Dubins, On Lebesgue-like extensions of finitely additive measures, Ann. Probability, 3 (1974), 456-463.

[3] Lester E. Dubins, and Leonard J. Savage, Inequalities for Stochastic Processes: How to Gamble if You Must, Dover, New York $(1965,1976)$.

[4] Lester E. Dubins and E. H. Spanier, How to cut a cake fairly, Amer. Math. Monthly, 68 (1961), 1-17.

[5] Lester E. Dubins, and William D. Sudderth, Countably additive gambling and optimal stopping, Z. Wahrscheinlichkeitstheorie verw. Gebiete, 41 (1977), 59-72.

[6] Victor C. Pestien, Stopping-time-indexed convergence and measurable gambling. Thesis, (1980), Department of Mathematics, University of California, Berkeley.

[7] Roger A. Purves, and William D. Sudderth, Some finitely additive probability, Ann. Probability, 4 (1976), 259-276.

[8] __ How to stay in a set or König's lemma for random paths, Israel J. Math., 43 (1982), 139-153.

[9] Ralph E. Stauch, Measurable gambling houses, Trans. Amer. Math. Soc., 126 (1976), 64-72. (correction 130, 184).

[10] William D. Sudderth, On measurable gambling problems, Ann. Math. Statist., 42 (1971), 260-269.

Received December 10, 1981 and in revised form September 17, 1982. 


\section{PACIFIC JOURNAL OF MATHEMATICS}

EDITORS

Donald BABBITt (Managing Editor)

University of California

Los Angeles, CA 90024

Hugo Rossi

University of Utah

Salt Lake City, UT 84112

C. C. Moore and Arthur Ogus

University of California

Berkeley, CA 94720

\section{J. DugundiI}

Department of Mathematics

University of Southern California

Los Angeles, CA 90089-1113

R. Finn and H. SAMELSON

Stanford University

Stanford, CA 94305

\section{ASSOCIATE EDITORS}
R. ARENS
E. F. BECKENBACH
B. H. NEUMANN
F. WOLF
K. YOSHIDA (1906-1982)

\section{SUPPORTING INSTITUTIONS}

UNIVERSITY OF ARIZONA

UNIVERSITY OF BRITISH COLUMBIA

CALIFORNIA INSTITUTE OF TECHNOLOGY

UNIVERSITY OF CALIFORNIA

MONTANA STATE UNIVERSITY

UNIVERSITY OF NEVADA. RENO

NEW MEXICO STATE UNIVERSITY

OREGON STATE UNIVERSITY
UNIVERSITY OF OREGION

UNIVERSITY OF SOUTHERN CALIFORNIA

STANFORD UNIVERSITY

UNIVERSITY OF HAWAII

UNIVERSITY OF TOKYO

UNIVERSITY OF UTAH

WASHINGTON STATE UNIVERSITY

UNIVERSITY OF WASHINGTON 


\section{Pacific Journal of Mathematics}

Vol. 109, No. $1 \quad$ May, 1983

Donald George Babbitt and V. S. Varadarajan, Formal reduction theory of meromorphic differential equations: a group theoretic view $\ldots \ldots \ldots \ldots 1$

Jo-Ann Deborah Cohen, Norms on $F(X) \ldots \ldots \ldots \ldots \ldots \ldots \ldots \ldots \ldots \ldots$

Robert Fitzgerald, Witt kernels of function field extensions $\ldots \ldots \ldots \ldots \ldots 89$

Hervé Jacquet and Joseph Andrew Shalika, The Whittaker models of induced representations .............................. 107

Masakiti Kinukawa, Some generalizations of contraction theorems for

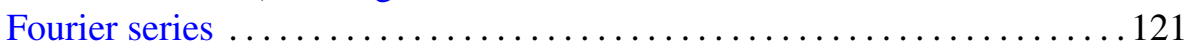

Joseph Weston Kitchen, Jr. and David A. Robbins, Sectional

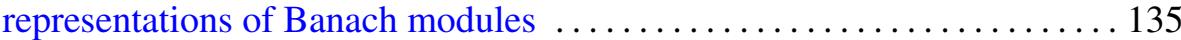

Victor Charles Pestien, Jr., Weak approximation of strategies in measurable gambling

Richard Scott Pierce and Charles Irvin Vinsonhaler, Realizing central

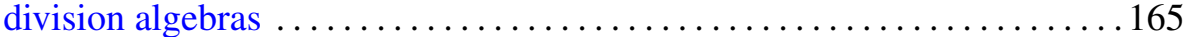

Walter Ricardo Ferrer Santos, Cohomology of comodules ............ 179

Marko Tadić, Harmonic analysis of spherical functions on reductive groups

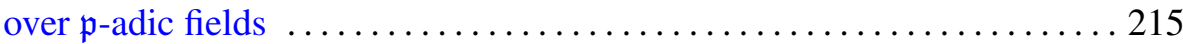

Lorenzo Traldi, The determinantal ideals of link modules. II . . . . . . . . 237

Alain J. Valette, A remark on the Kasparov groups $\operatorname{Ext}^{i}(A, B) \ldots \ldots \ldots 247$ 